\title{
Idiopathic non-cirrhotic portal hypertension: a review
}

\author{
Jeoffrey NL Schouten ${ }^{1}$, Joanne Verheij ${ }^{2}$ and Susana Seijo ${ }^{3^{*}}$
}

\begin{abstract}
Idiopathic non-cirrhotic portal hypertension (INCPH) is a rare disease characterized of intrahepatic portal hypertension in the absence of cirrhosis or other causes of liver disease and splanchnic venous thrombosis. The etiology of INCPH can be classified in five categories: 1) immunological disorders (i.e. association with common variable immunodeficiency syndrome, connective tissue diseases, Crohn's disease, etc.), 2) chronic infections, 3) exposure to medications or toxins (e.g. azathioprine, 6- thioguanine, arsenic), 4) genetic predisposition (i.e. familial aggregation and association with Adams-Oliver syndrome and Turner disease) and 5) prothrombotic conditions (e.g. inherited thrombophilias myeloproliferative neoplasm antiphospholipid syndrome). Roughly, INCPH diagnosis is based on clinical criteria and the formal exclusion of any other causes of portal hypertension. A formal diagnosis is based on the following criteria: 1) presence of unequivocal signs of portal hypertension, 2) absence of cirrhosis, advanced fibrosis or other causes of chronic liver diseases, and 3) absence of thrombosis of the hepatic veins or of the portal vein at imaging. Patients with INCPH usually present with signs or symptoms of portal hypertension such as gastro-esophageal varices, variceal bleeding or splenomegaly. Ascites and/or liver failure can occur in the context of precipitating factors. The development of portal vein thrombosis is common. Survival is manly limited by concomitant disorders. Currently, treatment of INCPH relies on the prevention of complications related to portal hypertension, following current guidelines of cirrhotic portal hypertension. No treatment has been studied aimed to modify the natural history of the disease. Anticoagulation therapy can be considered in patients who develop portal vein thrombosis.
\end{abstract}

Keywords: Non-cirrhotic portal hypertension, Portal hypertension, Variceal bleeding, Portal vein thrombosis

\section{Introduction}

Idiopathic non-cirrhotic portal hypertension (INCPH) is a rare disease characterized by of intrahepatic portal hypertension in the absence of cirrhosis, other causes of liver disease and splanchnic venous thrombosis [1-7]. Histological features of INCPH comprise a wide spectrum of nonspecific features, ranging from minor changes, sinusoidal dilatation, phlebosclerosis and portal fibrosis to nodular regenerative hyperplasia. It is still unclear whether this repertoire of histological changes reflects different stages of the disease, or it could also be that INCPH comprises different nosologic entities that share the same clinical presentation. Different conditions have been associated to this disorder including immune-based diseases, recurrent infections,

\footnotetext{
* Correspondence: susana.seijo@mssm.edu

${ }^{3}$ Department of Medicine, CTO, Icahn School of Medicine at Mount Sinai, New York, NY 10029, USA

Full list of author information is available at the end of the article
}

HIV infection and antiretroviral treatment, trace metals, certain medications and prothrombotic factors [1, 3, 7-9]. The pathophysiological mechanisms causing INCPH remain largely unknown. Patients with INCPH usually present with signs and symptoms of portal hypertension $(\mathrm{PH})$ such as splenomegaly, thrombocytopenia and variceal bleeding $[1,2,5]$. Patients can develop additional liver-related complications such as ascites, hepatic encephalopathy, portal vein thrombosis (PVT) or liver failure, that could eventually require liver transplantation (LT) $[2,3,6,10,11]$.

Orpha number: ORPHA64743

\section{Epidemiology \\ Although INCPH has a worldwide distribution, it is par- ticularly prevalent in Asia $[4,12,13]$. It is more frequent in socioeconomically disadvantaged individuals. Im- provements in hygiene and living standards may explain}

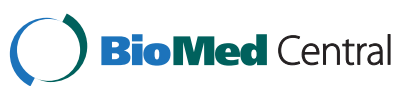

(C) 2015 Schouten et al. This is an Open Access article distributed under the terms of the Creative Commons Attribution License (http://creativecommons.org/licenses/by/4.0), which permits unrestricted use, distribution, and reproduction in any medium, provided the original work is properly credited. The Creative Commons Public Domain Dedication waiver (http:// creativecommons.org/publicdomain/zero/1.0/) applies to the data made available in this article, unless otherwise stated. 
the decreasing incidence of INCPH in Japan during the last decades, and the low prevalence of the disease in Western countries $[7,9,14,15]$. Gender and age disparities have also been reported $[4,15]$. In Western populations, median age at diagnosis is 40 years, with predominance in male gender. Conversely, Asian patients tend to be diagnosed at a younger age. In summary, differences in socioeconomic status, living conditions, pathogen exposure and ethnicity may play a role in INCPH development.

\section{Etiology and pathophysiology Etiology}

The etiology of INCPH is unknown $[1,4,9,14,16]$. Strikingly, small series and case studies show its association with an array of rare disorders; whether these associations are more than fortuitous remains unclear. Roughly, the potential mechanisms involved in INCPH pathogenesis can be classified in five main categories: immunological disorders, chronic infections, exposure to medications or toxins, genetic disorders and prothrombotic conditions (Table 1). A combination of these factors is also very likely. Associated conditions in 4 recently reported European series are described in Table 2 $[2,6,10,11,16]$.

\section{Immunological disorders}

In Western countries, INCPH is frequently associated with immunological disorders $[3,5,7,17,18]$. In patients with systemic sclerosis, enhanced fibrogenesis seems to have a major pathogenic role [8]. Also, in patients with

Table 1 Associated disorders of idiopathic non-cirrhotic portal hypertension

\begin{tabular}{ll}
\hline Immunological disorders & Common variable immunodeficiency \\
& syndrome [22, 54] \\
& Connective tissue diseases [55] \\
& Crohn's disease [26, 27] \\
& Solid organ transplant [56, 57] \\
& Bacterial intestinal infections [21] \\
& Human immunodeficiency virus (HIV) \\
Infections & infection [2, 9, 14, 22] \\
& Thiopurine derivatives (didanosine, \\
& azathioprine, cis-thioguanine) [27, 58] \\
& Arsenicals [28] \\
& Vitamin A [59] \\
& Adams-Olivier syndrome [32] \\
& Turner syndrome [30] \\
& Phosphomannose isomerase deficiency [60] \\
Genetic disorders & Familial cases [31, 61] \\
Inherited thrombophilias [2, 6, 10, 11, 62] & Myeloproliferative neoplasm [2, 6, 10, 11, 62] \\
Prothrombotic conditions & Antiphospholipid syndrome [2, 6, 10, 11, 62] \\
&
\end{tabular}

systemic lupus erythematosus, immunoglobulin interference with prostacyclin formation has been found to increase microthrombosis vulnerability [19]. In patients with celiac disease, an elevation of IgA anticardiolipin antibodies could be responsible for the obliteration of small vessels [1-7]. Interestingly, $70 \%$ of patients with primary hypogammaglobulinemia have histological features of INCPH [1, 3, 7-9, 20].

\section{Chronic infections}

Data indicate that intestinal infection with $E$. coli might cause recurrent septic embolization and subsequent obstruction of small portal veins, a probable trigger of INCPH. The high prevalence of INCPH in low socioeconomic areas with a high rate of abdominal infections in early childhood lends credit to this theory [1, 2, 4, 5]. In addition, experimental studies demonstrate how E. coli injection into the portal vein results in the development clinical and histological characteristics of INCPH $[2,3$, $6,10,11,21]$.

In Western countries, INCPH has been reported increasingly in patients with human immunodeficiency virus (HIV) infection [4, 9, 12-14, 17, 22-24]. Prolonged monotherapy or short-term combination treatment with didanosine and stavudine are independent risk factors for the development of this disorder, suggesting a potential role for mitochondrial toxicity in the development of INCPH [7, 9, 14, 15, 17, 22]. A recent multicenter study demonstrated a genetic predisposition to develop INCPH in HIV infected patients chronically exposed to didanosine $[4,15,25]$. Despite these data, it is difficult to assign a definitive etiopathogenic role of didanosine, as the drug has been widely used for the treatment of $\mathrm{HIV}$ in the past. Alternatively, a high prevalence of preexisting hypercoagulability, mainly due to protein $\mathrm{S}$ deficiency, possibly leading to vascular obstruction, has also been reported in patients with HIV-related INCPH [1, 4, $9,14,16]$. Hence, additional data will be needed to define a causal role of didanosine exposure and HIVassociated INCPH $[2,6,7,10,11,16,17]$.

\section{Exposure to medication and toxins}

Besides didanosine, exposure to other medications and chemicals has been reported to be associated with INCPH. Azathioprine, 6-thioguanine and arsenic as Fowler's solution are the most frequently reported drugs linked with this disorder [3, 5, 7, 17, 26-28]. Although it is tempting to blame drug intake and chemical exposure as primary etiological factors, only a very small proportion of the patients treated with the above mentioned drugs or exposed to these chemicals develop clinical or histological signs of INCPH. Clearly, additional factors may play a pathogenic role in these patients. 
Table 2 Associated conditions in 4 recently reported series of European patients with idiopathic non-cirrhotic portal hypertension

\begin{tabular}{|c|c|c|c|c|}
\hline Reference & Hillaire et al. [6] & Cazals-Hatem et al. [11] & Schouten et al. [10] & Siramolpiwat et al. [2] \\
\hline \multirow[t]{4}{*}{ Prothrombotic disorder } & 6 PS deficiency & 3 PS deficiency & 3 PS deficiency & 1 PS deficiency \\
\hline & 2 PC deficiency & 3 PC deficiency & 3 PC deficiency & 2 Fll Leiden \\
\hline & & 2 MTHFR mutation & 1 MTHFR mutation & 1 FV Leiden \\
\hline & & 3 Fll Leiden & 2 FV Leiden & \\
\hline Haematological malignancy & 1 & 0 & 4 & 5 \\
\hline Myeloproliferative neoplasm & 6 & 10 & 3 & 0 \\
\hline Chronic HIV infection & 0 & 0 & 5 & 15 \\
\hline Autoimmune disorder & 3 & 10 & 1 & 9 \\
\hline Crohn's disease & 0 & 0 & 3 & 0 \\
\hline Genetic disorder & 0 & 0 & 4 & 0 \\
\hline Solid organ malignancy & 0 & 0 & 1 & 0 \\
\hline Azathioprine treatment & 0 & 0 & 8 & 0 \\
\hline Arsenicals & - & 0 & 4 & 0 \\
\hline No associated conditions & 12 & 31 & 26 & 30 \\
\hline Incomplete evaluation & 2 & - & - & 9 \\
\hline Total $^{\mathrm{a}}$ & 28 & 59 & 62 & 69 \\
\hline
\end{tabular}

FII factor II, FV Leiden factor V Leiden, HIV human immunodeficiency virus, MTHFR metilentetrahydorfolate reductase, $P C$ protein C, PS protein S $(\mathrm{a})$ : Some patients have more than one associated condition

\section{Genetic disorders}

Reports on familial aggregation of INCPH and presence of its key histologic features in congenital disorders (e.g. Adams-Oliver syndrome, Turner disease) indicate a possible genetic component in INCPH [18, 29-33]. There is evidence of an association between HLA-DR3 and INCPH, what also supports an immunogenetic basis of this disorder [8, 31].

\section{Prothrombotic conditions}

Thrombophilia seems invariable behind some clinical and histologic features of patients with INCPH. There are different pieces of evidence suggestive of this including the high prevalence (30-50 \%) of pre-existing hypercoagulability commonly reported in INCPH patients [2, $6,10,11,19,34]$. Also, these patients tend to have a relatively high incidence of portal vein thrombosis $[2,6$, $10,11]$. In addition, certain pathologic features in liver specimens of INCPH patients support a dominant role of thrombophilia in the development of INCPH $[6,11$, $35,36]$. Presence of obliterative portal venopathy (i.e. luminal narrowing or obliteration of small portal venous branches accompanied by dense deposits of elastic fibers) in liver specimens of INCPH patients is highly suggestive of previous thrombotic episodes. Furthermore, the majority of liver explants from transplanted INCPH patients demonstrate organized old thrombi in the large portal vein branches [37, 38].

\section{Pathogenesis}

A dual theory, implicating both intrahepatic vascular obstruction and increased splanchnic blood flow, has been suggested to explain portal hypertension in INCPH patients $[1,6,39]$. An increased intrahepatic resistance likely results from the obstructed intrahepatic vessels (i.e. phlebosclerosis) and distorted intrahepatic angioarchitecture (i.e. nodular regeneration). The mechanisms responsible for the obliteration of portal venules remain unknown. Several hypotheses have been proposed [1, 34, 39], including aberrant coagulation activation or thrombosis, acquired or inherited disorders of vascular remodeling, and endothelial injury from immune cells [40]. Similar to cirrhosis, the imbalance of different vasoactive mediators causing intrahepatic vasoconstriction could also be considered. Additionally to the increased intrahepatic resistance, a portal venous overflow secondary to splenomegaly has been linked to the development of portal hypertension in INCPH patients $[41,42]$. Overproduction of nitric oxide, released in the sinus lining spleen cells, could also justify the dilatation of splenic sinuses and subsequent massive splenomegaly frequently found in INCPH patients $[1,43]$.

\section{Clinical manifestations}

Complications related to portal hypertension dominate the signs and symptoms present in patients with INCPH $[1,2,34,39]$. The liver function is usually preserved. 
Variceal bleeding is the most common clinical feature. Unlike cirrhotic patients, prognosis of variceal bleeding in INCPH is usually good due to the preserved liver function. In those patients without variceal bleeding at diagnosis, over $75 \%$ had varices at the initial endoscopy $[2,34]$. A recent study has shown that the 1 -year probability of developing small and large varices was $10 \%$ and $13 \%$, respectively; this is similar to what is described in cirrhotic patients [2]. This study also showed that in patients with large varices, the 1-year probability of first bleeding episode despite primary prophylaxis was $9 \%$. In addition, the 1 -year probability of re-bleeding despite combined secondary prophylaxis (i.e. betablockers and endoscopic band ligation) was $22 \%$ [2].

Ascites is reported in up to $50 \%$ of cases, and it usually develops in the context of precipitating factors such as variceal bleeding or infections. Generally, it is easily controlled with low dose of diuretics and resolution of the trigger $[1,10]$. Hepatic encephalopathy is a rare complication and it is also related to precipitating factors. There are anecdotic reports of hepatopulmonary syndrome, portopulmonary hypertension and hepatocellular carcinoma. Over $95 \%$ of patients have splenomegaly and it can cause left upper quadrant's abdominal pain.

Portal vein thrombosis (PVT) is also common, with a reported prevalence that ranges from 13-46\% $[2,6,10]$. A recent study found a $9 \%$ annual probability of developing PVT. HIV infection and the presence of variceal bleeding at diagnosis have been described as factors independently associated with a high risk of developing PVT $[2,7]$. Remarkably, most patients are asymptomatic at the time of PVT diagnosis. Therefore, it may be useful to screen for the presence of PVT in INCPH patients. It is unclear, however, the optimal frequency or best imaging modality in this context.

\section{Diagnosis}

There is a lack of a specific positive test that leads to an INCPH diagnosis. It is based on clinical criteria and the formal exclusion of other causes of $\mathrm{PH}$; this represents a clinical challenge, even in experienced liver units. Consequently, INCPH is frequently unrecognized, and in many instances patients are misdiagnosed with liver cirrhosis $[44,45]$.

\section{Criteria and differential diagnosis}

The diagnosis of INCPH is a diagnosis of exclusion, based on the following previously reported criteria [1]: 1) presence of unequivocal signs of portal hypertension (e.g., gastroesophageal varices, ascites, and/or splenomegaly); 2) absence of cirrhosis, advanced fibrosis or other causes of chronic liver diseases that can cause $\mathrm{PH}$ by appropriate serological, biochemical tests and liver biopsy and; 3) absence of thrombosis of the hepatic veins or of the portal vein at imaging studies performed at diagnosis.

Therefore, the current diagnostic work up for INCPH should include: 1) detailed medical history to investigate concomitant diseases and exposure to drugs, medications or toxins, 2) liver imaging to evaluate the patency of the splanchnic venous axis, 3) laboratory tests to rule out other causes of liver diseases and/or $\mathrm{PH}$ and 4) a mandatory liver biopsy to discard cirrhosis and other causes of chronic liver disease with or without PH. As a result, a diagnosis of INCPH can only be made upon the exclusion of liver cirrhosis, portal vein thrombosis, Budd-Chiari syndrome, chronic liver diseases causing noncirrhotic portal hypertension (e.g. chronic viral hepatitis, primary biliary cirrhosis, non-alcoholic steatohepatitis, alcoholic steatohepatitis and autoimmune hepatitis) and conditions causing portal hypertension (congenital liver fibrosis, sarcoidosis and schistomiasis).

\section{Liver function tests}

Liver function tests are usually within normal range; jaundice is rarely seen at diagnosis. Transient impairments in liver function may occur in the context of variceal bleeding or infection. Anemia, leukopenia, and thrombocytopenia are common due to hypersplenism.

\section{Imaging}

Comprehensive liver imaging is required before INCPH can be confidently diagnosed. The goals of liver imaging are twofold: 1) to determine the presence of radiological signs of PH such as splenomegaly, collaterals or ascites, and 2) to evaluate the patency of the hepatic veins and the porto-spleno-mesenteric venous axis. Of note, most patients also present radiological signs of chronic liver disease (i.e. liver surface nodularity) despite the lack of histologic cirrhosis [45]. Doppler ultrasound in addition to CT angiography or MRI angiography is the recommended strategy.

\section{Liver biopsy / liver pathology}

The morphological features associated with INCPH can be sometimes subtle, what makes pivotal an adequate histological evaluation by expert liver pathologists. It is essential to systematically assess the different anatomical structures in the liver, including their size, topography and morphology. At the portal tracts, presence of a bile duct, a branch of the hepatic artery and of a normally sized portal vein needs to be determined. Hypoplastic or minute portal tracts, with a lumen of the bile duct or artery smaller than the surrounding hepatocytes are typical of INCPH (Fig. 1) [35]. These are thought to be portal tract remnants, resulting from resorption of normal portal tract collagen [46]. Portal sclerosis or hepatoportal sclerosis is also a common feature. It consists of fibrous 

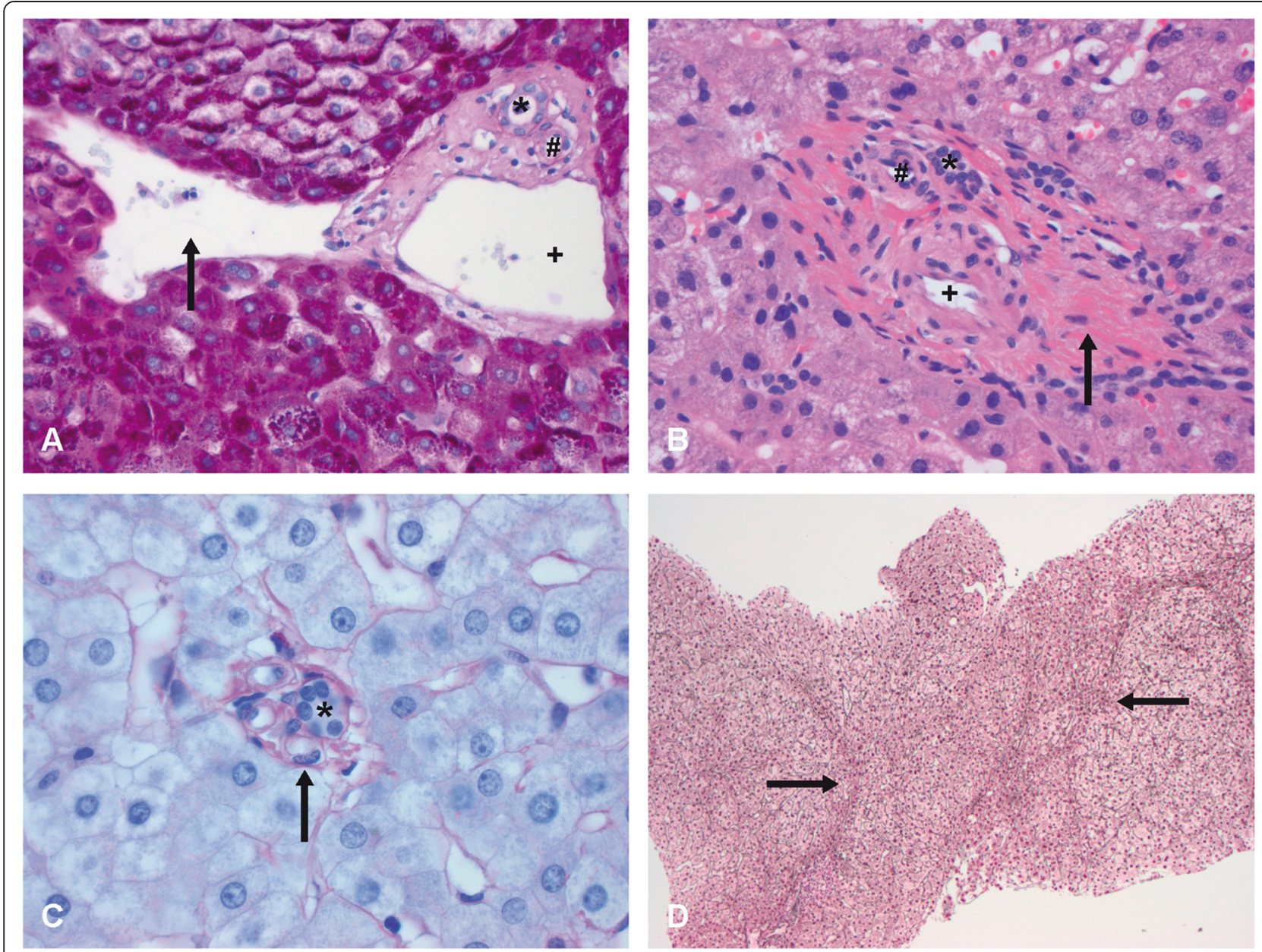

Fig. 1 a Paraportal shunting vessel (arrow), herniating into the liver parenchyma. The adjacent portal tract has a bile duct $\left({ }^{*}\right)$, hepatic artery (\#) and portal vein (+); PAS staining, 20x. b Phlebosclerosis. In a fibrotic portal tract (arrow), a bile duct $(*)$, hepatic artery (\#) and arterialised portal vein (+) are present; haematoxylin and eosin, 20x. c Hypoplastic portal tract in which the lumen of the bile duct $\left(^{*}\right)$ is smaller than the diameter of the surrounding hepatocytes; PAS-amylase staining, $40 \mathrm{x}$. $\mathbf{d}$ Nodular regenerative hyperplasia (NRH) with central hyperplasia and an atrophic rim (arrow) in the absence of fibrosis; reticulin staining, 10x

thickening of the portal vein wall and may result from portal vein thrombosis and consequent organization, either from the larger portal vein branches or in the context of local portal tract pathology. Some authors use the term obliterative portal venopathy for this entity. Other findings in the context of INCPH in the (peri)portal area include dilated portal veins, abnormal spacing between portal tracts and veins, an increased number of vascular structures in the portal tracts, arterialization of the wall of portal veins and the presence of paraportal shunting vessels and/or herniating in the liver parenchyma [38, 47, 48].

In the liver parenchyma, abnormalities that may be seen in the context of INCPH include sinusoidal dilatation, congestion and pericellular fibrosis, aberrant hepatic vessels and dilatation of the central vein with or without perivenular fibrosis. The lumen of the central veins can be (partially) occluded associated with different toxic agents. A regenerative, compensatory response can be the result of a heterogeneous blood flow in the presence of circulatory abnormalities at different levels of the microcirculation. This might lead to nodular regenerative hyperplasia, showing micronodular transformation, with central hyperplasia and an atrophic rim in the absence of fibrosis [48, 49]. Pathologists should become familiar with these features, in order to suggest or support the clinical diagnosis of INCPH in patients with non-cirrhotic portal hypertension.

\section{Other investigations}

$\mathrm{INCPH}$ is an intrahepatic presinusoidal cause of $\mathrm{PH}$ $[44,50]$. Hepatic venous pressure gradient (HVPG) is normal $(\leq 5 \mathrm{mmHg})$ or slightly increased $(5-10 \mathrm{mmHg})$ but below the previously described cut-off for clinically 
significant portal hypertension in cirrhosis (CSPH; HVPG $>10 \mathrm{mmHg}$ ) [37, 51]. Also, liver stiffness value on transient elastography (Fibroscan ${ }^{\circ}$ ) is lower than the described cut-off values for diagnosing cirrhosis, varices and CSPH $[2,7,51]$. Thus, lower values for HVPG and liver stiffness than those described for cirrhosis and $\mathrm{CSPH}$ can be helpful by ruling out cirrhosis in a patient with signs of PH.

\section{Treatment}

\section{Management of portal hypertension}

Data on management and prophylaxis of variceal bleeding in INCPH patients are scarce with a remarkable lack of randomized controlled trials. There are no specific guidelines for the management of $\mathrm{PH}$ in patients with INCPH. Nevertheless, expert opinion recommends following the guidelines of prophylaxis and management of $\mathrm{PH}$ in cirrhotic patients [1]. A recent cohort study reported good long-term outcome by applying a management strategy based on current guidelines for cirrhotic variceal bleeding [2].

Briefly, primary and secondary prevention of variceal bleeding includes the use of non-selective beta-blockers and endoscopic variceal ligation. Trans-jugular intrahepatic portosystemic shunting (TIPSS) is an effective alternative in patients who fail to respond to medical and endoscopic therapy. Management of acute variceal bleeding includes early pharmacological treatment with vasoactive drugs, early endoscopic control of bleeding, careful blood product replacement, and prophylactic antibiotics [52]. Guidelines also recommend withdrawing any drug potentially associated with INCPH (e.g. azathioprine) and treating any associated medical conditions [52].

\section{Liver transplant}

Even though INCPH patients usually have well preserved liver function, and $\mathrm{PH}$ related complications are successfully controlled, some patients may require a LT. Some of the reported indications for LT include unmanageable PH, progressive liver failure, chronic hepatic encephalopathy, hepatopulmonary syndrome and hepatocellular carcinoma. Post-LT outcomes of INCPH patients are good and the disease tends not to recur. However, data on this issue are limited and mostly based on small cohorts $[1,45]$.

\section{Management of PVT}

The use of anticoagulation in the management of PVT in INCPH is controversial, mainly due to the lack of prospective data. Nevertheless, we believe that anticoagulation therapy must be considered in patients with underlying prothrombotic conditions and in patients who develop PVT. A recent retrospective series described 15 patients with INCPH and PVT that were treated with anticoagulants. At the end of follow-up, $54 \%$ of patients achieved some degree of recanalization [2]. Regarding anticoagulation in these patients, some issues that need to be addressed: 1) which are the subgroup of PVT patients that benefit from anticoagulation, 2) which is the best anticoagulantion modality (low molecular weight heparins $v s$ vitamin-K antagonist $v s$ new oral anticoagulants), 3) which is the optimal duration of anticoagulation and 4) which are the early predictors of response. Another important point is whether anticoagulation may have a role in the prevention of PVT. Based on the high prevalence of thrombophilia, the frequent presence of thrombosis of small intrahepatic and main portal veins in INCPH, it would be even more important to determine whether anticoagulation could play a role to prevent disease progression.

\section{Prognosis}

Very few studies have evaluated the long-term prognosis of INCPH patients. Overall, prognosis is generally better than in patients with cirrhosis and a similar degree of portal hypertension. As mentioned above, this may be due to the fact that most INCPH patients have well preserved liver function. However, a small subgroup of patients will develop liver failure and will require LT. Two recent European cohort studies evaluated prognosis of INCPH $[2,10,32]$. The Dutch study reported low overall and LT-free survival, $78 \%$ and $72 \%$ at 5 years, respectively. However, it should be noted that only $13 \%$ of patients died from liver-related causes. Conversely, the Spanish cohort reported $86 \%$ of LT-free survival at 5 years. Interestingly, ascites was identified as a poor prognostic factor in both studies. The presence of a concomitant severe disorder such as an immunological disease or malignancy was also identified as a poor prognostic factor in the Spanish study.

\section{Conclusions and future perspectives}

Over the last decade, numerous efforts have tried to clarify different aspects of INCPH. First, the nomenclature concerning this clinical disorder has been ambiguous and highly depended on histological features. To facilitate future studies and subsequently enhance our understanding of the disease, common terminology and diagnostic criteria have been developed [1]. Regarding pathophysiology, some genetic traits have been identified in HIV-associated INCPH [25]. Furthermore, cohort studies performed in Europe provided new insights into the natural course and prognosis of these patients $[2,10$, 11]. Despite these improvements, several uncertainties related to its pathogenesis should be further addressed. Large multicenter studies studying INCPH prevalence, associated disorders, natural course and prognosis are an unmet need. The diagnosis of INCPH still relies on clinical and histologic elements; future research should 
provide diagnostic and prognostic biomarkers of the disease. Furthermore, no randomized controlled trials have been yet performed. Currently, treatment of INCPH patients is based on the prevention of complications of portal hypertension as per guidelines for patients with liver cirrhosis. These treatment modalities have not been systematically evaluated in INCPH. So far, no treatment able to modify the course of the disease or to prevent complications has been tested in INCPH. Considering the role of thrombophilia in the pathophysiology of this disorder, it seems that anticoagulation therapy could prevent the progression of INCPH, but prospective controlled data are still needed. Limited available data in INCPH patients show no increased risk of serious bleeding $[2,11,53]$ and a significant rate of portal vein recanalization in patients with associated portal vein thrombosis [2]. Furthermore, evidence generated in other vascular liver diseases such as PVT or Budd-Chiari syndrome demonstrate that prolonged anticoagulation improves outcomes without increasing significantly the risk of serious bleeding. Hopefully, future randomized trials will provide new tools to tackle this orphan disease and improve our understanding of its complex pathophysiology.

\section{Nomenclature \\ Idiopathic non-cirrhotic portal hypertension \\ non-cirrhotic portal fibrosis \\ hepatoportal sclerosis \\ incomplete septal cirrhosis \\ obliterative portal venopathy \\ partial nodular transformation}

\begin{abstract}
Abbreviations
$\mathrm{CSPH}$ : Clinically significant portal hypertension in cirrhosis; INCPH: Idiopathic non-cirrhotic portal hypertension; HIV: Human immunodeficiency virus; LT: Liver transplant; PH: Portal hypertension; PVT: Portal vein thrombosis; TIPS: Trans-jugular intrahepatic portosystemic shunting.
\end{abstract}

\section{Competing interests}

The authors declare that they have no competing interests.

\section{Authors' contributions}

All authors contributed to a draft of the manuscript and were subsequently involved in revising the manuscript critically for important intellectual content. All authors read and approved the final manuscript.

\section{Author details}

'Department of Gastroenterology, University Hospital Ghent, De Pintelaan 185, Ghent, Belgium. ²Department of Pathology, Academic Medical Center University of Amsterdam, Amsterdam, The Netherlands. ${ }^{3}$ Department of Medicine, CTO, Icahn School of Medicine at Mount Sinai, New York, NY 10029, USA

Received: 29 January 2015 Accepted: 20 May 2015

Published online: 30 May 2015

\section{References}

1. Schouten JNL, García-Pagán JC, Valla DC, Janssen HLA. Idiopathic noncirrhotic portal hypertension. Hepatology. 2011;54:1071-81.
2. Siramolpiwat S, Seijo S, Miquel R, Berzigotti A, García-Criado A, Darnell A, et al. Idiopathic portal hypertension: natural history and long-term outcome. Hepatology. 2014;59:2276-85

3. Eapen CE, Nightingale P, Hubscher SG, Lane PJ, Plant T, Velissaris D, et al. Non-cirrhotic intrahepatic portal hypertension: associated Gut diseases and prognostic factors. Dig Dis Sci. 2010;56:227-35.

4. Sarin SK, Kapoor D. Non-cirrhotic portal fibrosis: current concepts and management. J Gastroenterol Hepatol. 2002;17:526-34.

5. Austin A. Nodular regenerative hyperplasia of the liver and coeliac disease: potential role of IgA anticardiolipin antibody. Gut. 2004;53:1032-4.

6. Hillaire S, Bonte E, Denninger M-H, Casadevall N, Cadranel J-F, Lebrec D, et al. Idiopathic non-cirrhotic intrahepatic portal hypertension in the West: a re-evaluation in 28 patients. Gut. 2002;51:275-80.

7. Chang P-E, Miquel R, Blanco J-L, Laguno M, Bruguera M, Abraldes JG, et al. Idiopathic portal hypertension in patients with HIV infection treated with highly active antiretroviral therapy. Am J Gastroenterol. 2009;104:1707-14.

8. Tsuneyama K, Harada K, Katayanagi K, Watanabe K, Kurumaya H, Minato H, et al. Overlap of idiopathic portal hypertension and scleroderma: report of two autopsy cases and a review of literature. J Gastroenterol Hepatol. 2002;17:217-23.

9. Schouten JNL, Van der Ende ME, Koëter T, Rossing HHM, Komuta M, Verheij J, et al. Risk factors and outcome of HIV-associated idiopathic noncirrhotic portal hypertension. Aliment Pharmacol Ther. 2012;36(9):875-85.

10. Schouten JNL, Nevens F, Hansen B, Laleman W, den Born M, Komuta M, et al. Idiopathic noncirrhotic portal hypertension is associated with poor survival: results of a long-term cohort study. Aliment Pharmacol Ther. 2012;35:1424-33.

11. Cazals-Hatem D, Hillaire S, Rudler M, Plessier A, Paradis V, Condat B, et al. Obliterative portal venopathy: portal hypertension is not always present at diagnosis. J Hepatol. 2011;54:455-61.

12. Madhu K, Avinash B, Ramakrishna B, Eapen CE, Shyamkumar NK, Zachariah $U$, et al. Idiopathic non-cirrhotic intrahepatic portal hypertension: common cause of cryptogenic intrahepatic portal hypertension in a Southern Indian tertiary hospital. Indian J Gastroenterol. 2009;28:83-7.

13. Okudaira M, Ohbu M, Okuda K. Idiopathic portal hypertension and its pathology. Semin Liver Dis. 2002;22(1):59-72.

14. Mallet V, Blanchard P, Verkarre V, Vallet-Pichard A, Fontaine H, Lascoux-Combe C, et al. Nodular regenerative hyperplasia is a new cause of chronic liver disease in HIV-infected patients. AIDS. 2007;21:187-92.

15. Okuda K. Non-cirrhotic portal hypertension versus idiopathic portal hypertension. J Gastroenterol Hepatol. 2002;17 Suppl 3:S204-13.

16. Mallet VO, Varthaman A, Lasne D, Viard J-P, Gouya H, Borgel D, et al. Acquired protein $\mathrm{S}$ deficiency leads to obliterative portal venopathy and to compensatory nodular regenerative hyperplasia in HIV-infected patients. AIDS. 2009;23:1511-8

17. Vispo E, Moreno A, Maida I, Barreiro P, Cuevas A, Albertos S, et al. Noncirrhotic portal hypertension in HIV-infected patients: unique clinical and pathological findings. AIDS. 2010;24:1171-6.

18. Umeyama K, Yui S, Fukamizu A, Yoshikawa K, Yamashita T. Idiopathic portal hypertension associated with progressive systemic sclerosis. Am J Gastroenterol. 1982;77:645-8.

19. Carreras LO, Defreyn G, Machin SJ, Vermylen J, Deman R, Spitz B, et al. Arterial thrombosis, intrauterine death and "lupus" antiocoagulant: detection of immunoglobulin interfering with prostacyclin formation. Lancet. 1981;1:244-6.

20. Malamut G, Ziol M, Suarez F, Beaugrand M, Viallard JF, Lascaux AS, et al. Nodular regenerative hyperplasia: the main liver disease in patients with primary hypogammaglobulinemia and hepatic abnormalities. J Hepatol. 2008:48:74-82.

21. Kono K, Ohnishi K, Omata M, Saito M, Nakayama T, Hatano H, et al. Experimental portal fibrosis produced by intraportal injection of killed nonpathogenic Escherichia coli in rabbits. Gastroenterology. 1988;94:787-96.

22. Maida I, Garcia-Gasco P, Sotgiu G, Ríos MJ, Vispo ME, Martín-Carbonero L, et al. Antiretroviral-associated portal hypertension: a new clinical condition? Prevalence, predictors and outcome. Antivir Ther. 2008;13:103-7.

23. Saifee S, Joelson D, Braude J, Shrestha R, Johnson M, Sellers M, et al. Noncirrhotic portal hypertension in patients with human immunodeficiency virus-1 infection. Clin Gastroenterol Hepatol. 2008;6:1167-9.

24. Martinez-Palli G, Drake BB, García-Pagán JC, Barbera J-A, Arguedas MR, Rodriguez-Roisin R, et al. Effect of transjugular intrahepatic portosystemic shunt on pulmonary gas exchange in patients with portal hypertension and hepatopulmonary syndrome. World J Gastroenterol. 2005;11:6858-62. 
25. Vispo E, Cevik M, Rockstroh JK, Barreiro P, Nelson M, Scourfield A, et al. Genetic determinants of idiopathic noncirrhotic portal hypertension in HIV-infected patients. Clin Infect Dis. 2013;56:1117-22.

26. de Boer NKH, Tuynman H, Bloemena E, Westerga J, Van Der Peet DL, Mulder CJJ, et al. Histopathology of liver biopsies from a thiopurine-naïve inflammatory bowel disease cohort: prevalence of nodular regenerative hyperplasia. Scand J Gastroenterol. 2008;43:604-8.

27. Vernier-Massouille G, Cosnes J, Lemann M, Marteau P, Reinisch W, Laharie D, et al. Nodular regenerative hyperplasia in patients with inflammatory bowel disease treated with azathioprine. Gut. 2007;56:1404-9.

28. Nevens F, Fevery J, Van Steenbergen W, Sciot R, Desmet V, De Groote J. Arsenic and non-cirrhotic portal hypertension. A report of eight cases. J Hepatol. 1990;11:80-5.

29. Moran CA, Mullick FG, Ishak KG. Nodular regenerative hyperplasia of the liver in children. Am J Surg Pathol. 1991;15:449-54

30. Roulot D. Liver involvement in Turner syndrome. Liver Int. 2012;33:24-30

31. Sarin SK, Mehra NK, Agarwal A, Malhotra V, Anand BS, Taneja V. Familial aggregation in noncirrhotic portal fibrosis: a report of four families. Am J Gastroenterol. 1987:82:1130-3.

32. Girard M, Amiel J, Fabre M, Pariente D, Lyonnet S, Jacquemin E. Adams-Oliver syndrome and hepatoportal sclerosis: occasional association or common mechanism? Am J Med Genet. 2005;135A:186-9.

33. Barnett $J$, Appelman HD, Moseley RH. A familial form of incomplete septa cirrhosis. Gastroenterology. 1992;102:674-8.

34. Sarin SK, Khanna R. Non-cirrhotic portal hypertension. Clin Liver Dis. 2014;18:451-76.

35. Verheij J, Schouten JNL, Komuta M, Nevens F, Hansen BE, Janssen HLA, et al. Histological features in western patients with idiopathic non-cirrhotic portal hypertension. Histopathology. 2013;62:1083-91.

36. Wanless IR, Bernier V, Seger M. Intrahepatic portal vein sclerosis in patients without a history of liver disease. Am J Pathol. 1982;106(1):63-70.

37. Okuda K, Kono K, Ohnishi K, Kimura K, Omata M, Koen H, et al. Clinical study of eighty-six cases of idiopathic portal hypertension and comparison with cirrhosis with splenomegaly. Gastroenterology. 1984;86:600-10.

38. Nakanuma Y, Hoso M, Sasaki M, Terada T, Katayanagi K, Nonomura A, et al. Histopathology of the liver in non-cirrhotic portal hypertension of unknown aetiology. Histopathology. 1996;28:195-204.

39. Khanna R, Sarin SK. Non-cirrhotic portal hypertension - Diagnosis and management. J Hepatol. 2014;60:421-41.

40. Ziol M, Poirel H, Kountchou GN, Boyer O, Mohand D, Mouthon L, et al. Intrasinusoidal cytotoxic CD8+ T cells in nodular regenerative hyperplasia of the liver. Hum Pathol. 2004:35:1241-51.

41. Ohnishi K, Saito M, Sato S, Terabayashi H, lida S, Nomura F, et al. Portal hemodynamics in idiopathic portal hypertension (Banti's syndrome). Comparison with chronic persistent hepatitis and normal subjects. Gastroenterology. 1987;92:751-8.

42. Blendis LM, Banks DC, Ramboer C, Williams R. Spleen blood flow and splanchnic haemodynamics in blood dyscrasia and other splenomegalies. Clin Sci. 1970;38:73-84

43. Sato Y, Sawada S, Kozaka K, Harada K, Sasaki M, Matsui O, et al. Significance of enhanced expression of nitric oxide syntheses in splenic sinus lining cells in altered portal hemodynamics of idiopathic portal hypertension. Dig Dis Sci. 2007:52:1987-94

44. Berzigotti A, Seijo S, Reverter E, Bosch J. Assessing portal hypertension in liver diseases. Expert Rev Gastroenterol Hepatol. 2013:7:141-55.

45. Krasinskas AM, Eghtesad B, Kamath PS, Demetris AJ, Abraham SC. Liver transplantation for severe intrahepatic noncirrhotic portal hypertension. Liver Transpl. 2005;11:627-34.

46. Wanless IR, Nakashima E, Sherman M. Regression of human cirrhosis. Morphologic features and the genesis of incomplete septal cirrhosis. Arch Pathol Lab Med. 2000;124:1599-607.

47. Verrijken A, Francque S, Mertens I, Prawitt J, Caron S, Hubens G, et al. Prothrombotic factors in histologically proven nonalcoholic fatty liver disease and nonalcoholic steatohepatitis. Hepatology. 2013:59:121-9.

48. Bioulac-Sage P, Le Bail B, Bernard PH, Balabaud C. Hepatoportal sclerosis. Semin Liver Dis. 1995:15:329-39.

49. Wanless IR. Micronodular transformation (nodular regenerative hyperplasia) of the liver: a report of 64 cases among 2500 autopsies and a new classification of benign hepatocellular nodules. Hepatology. 1990;11:787-97.
50. Sarin SK, Sethi KK, Nanda R. Measurement and correlation of wedged hepatic, intrahepatic, intrasplenic and intravariceal pressures in patients with cirrhosis of liver and non-cirrhotic portal fibrosis. Gut. 1987;28(3):260-6.

51. Seijo S, Reverter E, Miquel R, Berzigotti A, Abraldes JG, Bosch J, et al. Role of hepatic vein catheterisation and transient elastography in the diagnosis of idiopathic portal hypertension. Dig Liver Dis. 2012;44:855-60.

52. Chang P-E, Garcia-Pagan J. Causes of Noncirrhotic Portal Hypertension. Up to date: 2014:1-28.

53. Bihl F, Janssens F, Boehlen F, Rubbia-Brandt L, Hadengue A, Spahr L. Anticoagulant therapy for nodular regenerative hyperplasia in a HIV-infected patient. BMC Gastroenterol. 2010;10:6.

54. Fuss IJ, Friend J, Yang Z, He JP, Hooda L, Boyer J, et al. Nodular regenerative hyperplasia in common variable immunodeficiency. J Clin Immunol. 2013;33:748-58.

55. Vaiphei K, Bhatia A, Sinha SK. Liver pathology in collagen vascular disorders highlighting the vascular changes within portal tracts. Indian J Pathol Microbiol. 2011:54:25-31.

56. Allison MC, Mowat A, McCruden EA, McGregor E, Burt AD, Briggs JD, et al. The spectrum of chronic liver disease in renal transplant recipients. Q J Med. 1992;83:355-67.

57. Gane E, Portmann B, Saxena R, Wong P, Ramage J, Williams R. Nodular regenerative hyperplasia of the liver graft after liver transplantation. Hepatology. 1994;20:88-94

58. Calabrese E, Hanauer SB. Assessment of non-cirrhotic portal hypertension associated with thiopurine therapy in inflammatory bowel disease. J Crohns Colitis. 2011:5:48-53.

59. Geubel AP, De Galocsy C, Alves N, Rahier J, Dive C. Liver damage caused by therapeutic vitamin A administration: estimate of dose-related toxicity in 41 cases. Gastroenterology. 1991;100:1701-9.

60. de Lonlay $\mathrm{P}$, Seta $\mathrm{N}$. The clinical spectrum of phosphomannose isomerase deficiency, with an evaluation of mannose treatment for CDG-Ib. Biochim Biophys Acta. 1792;2009:841-3.

61. Franchi-Abella S, Fabre M, Mselati E, De Marsillac ME, Bayari M, Pariente D, et al. Obliterative portal venopathy: a study of 48 children. J Pediatr. 2014:165:190-3. e2.

62. Bayan K, Tüzün Y, Yılmaz Ş, Canoruc N, Dursun M. Analysis of inherited thrombophilic mutations and natural anticoagulant deficiency in patients with idiopathic portal hypertension. J Thromb Thrombolysis. 2008;28:57-62.

\section{Submit your next manuscript to BioMed Central and take full advantage of:}

- Convenient online submission

- Thorough peer review

- No space constraints or color figure charges

- Immediate publication on acceptance

- Inclusion in PubMed, CAS, Scopus and Google Scholar

- Research which is freely available for redistribution 